\title{
Media Reviews
}

Review of Brought to Life: Exploring the History of Medicine, Science Museum, London, www.sciencemuseum.org.uk/broughttolife.aspx

There is an increasing amount of content relating to the history of medicine appearing online and many of our journals are adding 'media' or 'digital humanities' review sections. Yet, unlike book reviews, it remains unclear how scholars should go about writing a peerreview of a website. This review of Brought to Life: Exploring the History of Medicine makes an attempt to outline the criteria by which websites could be reviewed.

Like books, websites should be reviewed based on their content, readability and argument. Yet, unlike books, websites should also be reviewed based on their design and accessibility. To evaluate Brought to Life, this reviewer took into account the purpose, argument, content, sources, usability and navigation of the site in an attempt to satisfy the simultaneous need to assess the accessibility of the content and the merit of the content itself.

Brought to Life is a multimedia website that presents images of objects from the medical collection at the Science Museum, London. The purpose of the site is to create a 'valuable resource for teachers and students working on the history of medicine and related subjects' while also 'engaging people of all ages and interests in the history of medicine'. This sense of purpose is shared by many other informational and educational websites and, by translating the wealth of information and objects located at the Science Museum, London into an online space, Brought to Life creates a valuable collection of digital media that has the potential to have a strong impact on a variety of users.

An implicit argument of the site is that users can employ objects as a means to teach the history of medicine and trace techniques and treatments over time. The content on the site is divided up into sixteen themes, which emphasise factors influencing change and continuity over time and aim to support students who are studying the 'Medicine Through Time' GCSE History module. The way the themes are delineated is not only for teachers and students of the GCSE History module, but is also meant to engage students in undergraduate courses as well as seasoned scholars. To aid this variety of users, the site has introductions to each theme with key people, objects and places linked out to other pages.

Each of the sixteen themes is broken down into five subtopics and each subtopic is associated with objects relating to that topic. For example, a theme titled 'Controversies and Medicine' is associated with an image of the guillotine blade that decapitated JeanBaptiste Carrier, and the theme 'Public Health' is associated with an image of an influenza test kit from 1986. Users can access objects not only through the theme they are associated with but also by navigating to the 'objects' tab. When a user clicks on the 'objects' tab, they can navigate to objects by selecting a 'theme' or 'place' from a drop-down list.

Objects are also associated with other objects outside of their theme. This is helpful for browsing the collection, but at times the associations are tangential. For example, Professor Matthew Hay's academic cap, gown and hood from 1927 are associated with a mid-nineteenth century amulet from India. In general, the tactic of associating objects with other objects benefits the user because it allows users to chart a path through the objects by following their innate interest rather than any particular narrative. Yet, here it 
would have been nice to see more clear associations between the objects, which could have hinged on material, purpose, time and place.

In addition to 'Objects' and 'Themes', the site content includes 'People' and 'Techniques and Technologies'. When a user clicks on 'Techniques and Technologies' they are brought to a page that presents a window, which allows users to scroll through a list of techniques - such as amputation and trephination - alphabetically. Below the window are four drop-down lists arranged alphabetically. Similarly, the 'People' page has a timeline and below that timeline is a set of drop-down lists. One major downside to the site is the use of these drop-down lists, which make the site hard to navigate. Each dropdown list includes a portion of the alphabet and sometimes hundreds of options, which makes the content seem unmanageable.

The site also has a number of interactive graphics, which are sometimes very successful in advancing the goals of the site and sometimes not so successful. 'Preventing Infection in Hospitals' prompts users to interact with hypothetical scenarios which illustrate that from the 1950s through to the present medical professionals have had to walk a delicate line between keeping the hospital germ free and caring for patients. The graphics of this particular interactive portion are very crude.

However, other interactive portions of the site are more successful. One particularly engaging graphic was 'The Virtual Patient'. This animation allows the user to pick one of three patients and see how a physician would have treated the patient in the four eras: Ancient Greece, Medieval, 1700s and 1950s. Similarly, the graphic 'The Right Tool for the Job' allows users to select a tool and match it to a patient. For teenage users studying the history of medicine these graphics could be quite engaging because they present the material in a fun (if somewhat gross!) way.

Users can save any text or image to a portion of the site titled 'my collection' and can create quizzes or documents. The quizzes portion of the site allows educators to create image- or text-based quizzes and share them with students. This is a wonderful feature for instructors and would likely engage students with the site itself. Users can add items to a collection to view later and create an account that allows them to store these items - an incredibly valuable feature for everyone.

The authors of the material in Brought to Life are tucked away in a 'thank you' page in the 'about us' section of the site and the material on the site does not give credit to authors. Unlike books, which generally provide clear authorship, websites frequently conceal authorship. Perhaps as more websites are reviewed as pieces of produced knowledge rather than as teaching tools alone scholars will be given authorship. Despite being opaque about the content creators, the sources used are transparent and are listed at the bottom of each page. This is not only helpful for providing further reading, but also gives further opportunity for a scholarly dialogue about the site.

Presenting a full account of all the features or content in Brought to Life is a near impossible task given that the site details more than 2500 objects that are used to illustrate the history of medicine over a large span of time and place. What made reviewing the website even harder was that much of the content was unavailable during the time it was being reviewed. For example, neither the 'people timeline' nor the general timeline would load, the introductions to each theme were unavailable and the glossary items were unavailable. A large portion of the content had to be accessed via an archived version through archive.org. This raises a set of questions that many historians have had about reviewing websites; primarily, how can historians review something that is not static. 
I believe that there is a space for reviewing websites in journals, but that some digital material would be better served to be reviewed online where there is a greater opportunity for dialogue between reviewer and producer.

Heidi Knoblauch

Yale University, USA

doi: $10.1017 / \mathrm{mdh} .2013 .58$

Review of Contagion: Historical Views of Diseases and Epidemics, Harvard University, http://ocp.hul.harvard.edu/contagion/

In the early morning of 14 November 1721, someone threw a homemade bomb through the window of Cotton Mather's home in Boston. This threat on Mather's life was in protest of his support for the practice of inoculation (a note attached to the bomb read 'Cotton Mather, You Dog, Dam You: I'll inoculate you with this, with a Pox to you'). ${ }^{1}$ At the time, the city was in the midst of a smallpox epidemic that eventually resulted in nearly 6000 people contracting the disease and 844 deaths. In this context, the use of inoculation generated much controversy and gave rise to a minor pamphlet war over its moral and medical implications. These pamphlets and many other primary source materials related to this and other key episodes in the history of disease since 1500 are now available online at Contagion: Historical Views on Diseases and Epidemics, a website and digital library collection developed and hosted by Harvard University Library's Open Collections Program. This unique online collection of materials from several repositories at Harvard University includes 'digitized copies of books, serials, pamphlets, incunabula, and manuscripts' from the first outbreak of syphilis in Europe in 1494 to the founding of the World Health Organisation (WHO) in 1948. As indicated on the homepage, the developers of Contagion hope that the site will be useful not just to students and scholars of the history of medicine but also to 'researchers seeking a historical context for current epidemiology'.

While its primary function is to serve as a portal to this digital library collection, Contagion is also an online exhibit that tells the history of disease in the modern world through the stories of several diseases and epidemics since 1500, mostly in Western Europe and the US. While several pages of the exhibit focus on specific epidemics including 'The Great Plague of London, 1665', 'The Boston Smallpox Epidemic, 1721', “'Spanish' Influenza in North America, 1918-1919”' and 'The Yellow Fever Epidemic in Philadelphia, 1793', other pages provide a broader perspective on a specific disease with titles such as 'Cholera Epidemics in the 19th Century', "'Pestilence' and the Printed Books of the Late 15th Century", 'Syphilis, 1494-1923', 'Tropical Diseases and the Construction of the Panama Canal, 1904-1914' and 'Tuberculosis in Europe and North America, 1800-1922'. In these sections of the site, the specific diseases and epidemics are also used to highlight broader themes and concepts in the history of medicine. The site also offers additional 'explanatory pages that introduce concepts related to diseases and epidemics' with topics such as 'Colonialism and International Medicine',

\footnotetext{
${ }^{1}$ Quoted in Cristobal Silva, Miraculous Plagues: An Epidemiology of Early New England Narrative (Oxford: Oxford University Press, 2011), p. 218, n. 38.
} 